\title{
Global Stability For Double-Diffusive Convection In A Couple-Stress Fluid Saturating A Porous Medium
}

https://doi.org/10.2478/sgem-2018-0044

received July 6, 2018; accepted December 3, 2018.

Abstract: We show that the global non-linear stability threshold for convection in a double-diffusive couplestress fluid saturating a porous medium is exactly the same as the linear instability boundary. The optimal result is important because it shows that linearized instability theory has captured completely the physics of the onset of convection. It is also found that couple-stress fluid saturating a porous medium is thermally more stable than the ordinary viscous fluid, and the effects of couple-stress parameter $(F)$, solute gradient $\left(S_{f}\right)$ and Brinkman number $\left(D_{a}\right)$ on the onset of convection is also analyzed.

Keywords: couple-stress parameter, solute gradient, Brinkman number.

\section{Introduction}

Conventional hydrodynamic stability theory is mainly concerned with the determination of critical values of Rayleigh number, demarcating a region of stability from that of instability. The potentials of linear theory of stability and the energy method are complementary to each other in the sense that the linear theory gives conditions under which the hydrodynamic system is definitely unstable. It cannot with certainty conclude stability. On the other hand, the energy theory gives conditions under which the hydrodynamic system is definitely stable. It cannot with certainty conclude instability. Suffering from its basic assumptions, the validity of the linearized stability theory becomes questionable.

Hence, the non-linear approach becomes inevitable to investigate the effect of finite disturbances. The

*Corresponding author: Shalu Choudhary, Department of Mathematics, Uttaranchal University, Dehradun, Uttarakhand 248007, India, E-mail: chaudhary.shalu40@gmail.com

Sunil: Department of Mathematics, National Institute of Technology, Hamirpur, Himachal Pradesh 177005, India formulation and derivation of the basic equation of a layer of fluid heated and soluted from below in the porous medium using Boussinesq approximation has been given in a treatise by Joseph [1]. When a fluid flows in an isotropic and homogenous porous medium, the gross effect is represented by the Darcy's law. The study of a layer of fluid heated and soluted from below in the porous media is motivated both theoretically and by its practical application in engineering. Among the application in engineering disciplines, one can find the food process industry, chemical process industry and solidification and centrifugal casting of metals. The oldest method of non-linear stability analysis that can deal with finite disturbances is the energy method, originated by Orr [2], and its recent revival has been inspired by the work of Serrin [3] and Joseph [1, 4, 5]. Rapid improvements of the classical energy theory have been made in recent years [6]. The approach adopted in the present article is by the application of the energy method, pioneered and developed in its modern use way by Straughan [7, 8]. Straughan [9] developed a sharp non-linear energy stability analysis for the saturated porous medium, and the results obtained are the best possible showing that subcritical instabilities are not possible. By selecting the optimal, it has been possible to sharpen the stability bound in many physical problems (Straughan [8]). A nonlinear stability analysis of fluids by using generalized energy stability theory has been considered by many authors[10-15]).

There are a lot of analyses of performance and experiment in the couple-stress lubricant. Stokes [16] proposed a simplest theory called the Stokes microcontinum theory, which could be used for the simulation of couple-stress fluid. One of the applications of couple-stress fluid is its use in the study of the mechanism of lubrication of synovial joints, which has become the object of scientific research. A human joint is a dynamically loaded bearing that has auricular cartilage as the bearing and synovial fluid as the lubricant. When a fluid film is generated, squeeze-film action is capable of providing considerable protection to the cartilage surface. Ramanaian [17] applied the couple-stress fluid model 
to analyze the long slider bearing. Sharma and Thakur [18] and Sharma et al. [19] have studied the problems of couple-stress fluid heated and soluted from below in the hydromagnetic porous medium and rotation separately. Recently, Sunil and Mahajan [20-23] studied the non-linear stability analysis for thermal convection in a magnetized ferrofluid heated from below saturating a porous medium. Sunil et al. [24, 25] studied the non-linear stability analysis for thermal convection in a couple-stress fluid heated from below saturating a porous medium. Hsu et al. [26] studied the combined effects of couple stress and surface roughness using journal bearings lubricated with the nonNewtonian fluid. It was found that the combined effects of couple stress and surface roughness can improve the load carrying capacity and decrease the attitude angle and friction parameter. Recently, Lahmar [27] also found that the lubricants with couple-stress fluid would increase the load carrying capacity and stability and decrease the friction factor and the attitude angle.

The purpose of the present article was to study the non-linear stability analysis of couple-stress fluid heated and soluted from below, saturating a porous medium of high permeability [28]. The really interesting situation from a mathematical viewpoint arises when the layer is simultaneously heated and salted from below. In the standard Bènard problem, the instability is driven by a density difference caused by a temperature difference between the upper and lower surfaces bounding the fluid. If, additionally, the fluid layer has salt dissolved in it, then there are potentially two destabilizing sources for the density difference, the temperature field and the salt field. When there are two effects such as this, the phenomenon of convection that arises is called doublediffusive convection. The driving force for many studies in double-diffusive or multicomponent convection is largely physical applications. The double-diffusive convection problems have been studied by many authors [13-14, 29-39]. In porous media, an alternative to Darcy's equation is what is known as Brinkman's equation [28]. It is believed that for the flow of a high-porosity porous medium, the Brinkman equation removes some of the deficiencies and gives preferable result. In the work of Qin and Kaloni [40], it was remarked that for high porosity materials and when boundary layer effects need to be taken into account, the Brinkman model is superior to Darcy's model. Here, we establish the optimal result, that is the linear instability and non-linear stability of Rayleigh numbers are the same, i.e. $R_{\ell f} \equiv R_{e f}$. We also find that the critical value of thermal Rayleigh number for the couple-stress fluid is higher than the critical value of thermal Rayleigh number for the ordinary fluid; hence,

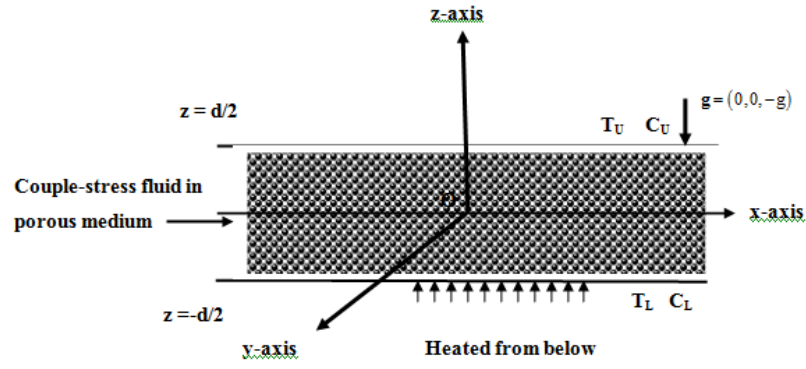

Figure 1: Geometrical configuration of the problem.

the couple-stress fluid is thermally more stable than the ordinary fluid. This problem, to the best of my knowledge, has not been investigated yet.

\section{Mathematical Formulation Of The Problem}

Here, we consider an infinite, horizontal layer of thickness ' $d$ ' of incompressible thin couple-stress fluid with constant viscosity that is heated and soluted from below, saturating an isotropic homogeneous porous medium of porosity $\varepsilon$ and medium permeability $K_{1}$.

The fluid is assumed to occupy the layer $z \in\left(-\frac{d}{2}, \frac{d}{2}\right)$ with gravity field $\mathbf{g}=(0,0,-g)$ pervading the system in the negative $z$-direction.

The equations governing the flow of an incompressible couple-stress fluid (utilizing the Boussinesq approximation) are given as follows [13, 24, 41]:

Mass balance:

$$
\nabla \cdot \mathbf{q}=0
$$

Momentum balance:

$\frac{\rho_{0}}{\varepsilon}\left[\frac{\partial \mathbf{q}}{\partial t}\right]=-\nabla p+\rho_{0}\left[1-\alpha\left(T-T_{a}\right)+\alpha^{\prime}\left(C-C_{a}\right)\right] \mathbf{g}-\frac{1}{K_{1}}\left(\mu-\mu^{\prime} \nabla^{2}\right) \mathbf{q}+\tilde{\mu} \nabla^{2} \mathbf{q}$.

Temperature equation:

$$
\left(\rho_{0} C_{0}\right)_{m} \frac{\partial T}{\partial t}+\left(\rho_{0} C_{0}\right)_{f} \mathbf{q} \cdot \nabla T=\nabla \cdot(k \nabla T) .
$$

Solute equation:

$$
\left(\rho_{0} C_{0}\right)_{m} \frac{\partial C}{\partial t}+\left(\rho_{0} C_{0}\right)_{f} \mathbf{q} \cdot \nabla C=\nabla \cdot\left(k^{\prime} \nabla C\right) .
$$

Here $\rho, \rho_{0}, \mathbf{q}, \mathbf{g}, t, p, \mu, \mu^{\prime}, \tilde{\mu}, \kappa, \kappa^{\prime}, K_{1}$, $\varepsilon, \alpha, \alpha^{\prime}$ and $C_{0}$ are the fluid density, reference density, filter velocity, acceleration due to gravity, time, pressure, coefficient of viscosity, coefficient of 
visco-elasticity, effective viscosity, thermal diffusivity, solute diffusivity, permeability of porous medium, porosity, thermal expansion coefficient, concentration expansion coefficient analogous to the thermal expansion coefficient and specific heat at constant pressure, respectively. The subscripts ' $m$ ' and ' $f$ ' refer to the fluidsolid mixture and the fluid, respectively. $T_{a}$ and $C_{a}$ are the average temperature and solute concentration given by $T_{a}=\frac{\left(T_{L}+T_{U}\right)}{2}$ and $C_{a}=\frac{\left(C_{L}+C_{U}\right)}{2}$ respectively, where $T_{L}$, $T_{U}$ and $C_{L}^{2}, C_{U}$ are the constant average temperatures and solute concentrations of the lower and upper surfaces of the layer, respectively, and $\beta\left(=\frac{d T}{d z}\right)$ and $\beta^{\prime}\left(=\frac{d C}{d z} \mid\right)$ are uniform temperature and solute gradients, respectively.

The basic state is assumed to be the quiescent state and is given by

$$
\begin{gathered}
\mathbf{q}=\mathbf{q}_{b}=(0,0,0), p=p_{b}(z), \\
\rho=\rho_{b}(z)=\rho_{0}\left(1+\alpha \beta z-\alpha^{\prime} \beta^{\prime} z\right), T=T_{b}(z)=-\beta z+T_{a}, \\
C=C_{b}(z)=-\beta^{\prime} z+C_{a}, \beta=\frac{T_{L}-T_{U}}{d}, \beta^{\prime}=\frac{C_{L}-C_{U}}{d},
\end{gathered}
$$

where the subscript ' $b$ ' denotes the basic state.

We shall analyze the stability of the basic state by introducing the following perturbations:

$$
\begin{gathered}
\mathbf{q}=\mathbf{q}_{b}+\mathbf{q}^{\prime}, \rho=\rho_{b}+\rho^{\prime}, p=p_{b}(z)+p^{\prime}, T=T_{b}(z)+\theta \text { and } \\
C=C_{b}(z)+\gamma .
\end{gathered}
$$

The non-linear equations for the perturbations $\mathbf{q}^{\prime}=(u, v, w), p^{\prime}, \rho^{\prime}, \theta$ and $\quad \gamma$, which represent perturbations in velocity $\mathbf{q}$, pressure $p$, density $\rho$, temperature $T$ and concentration $C$, respectively, are given by

$$
\begin{aligned}
\frac{\rho_{0}}{\varepsilon} \frac{\partial \mathbf{q}^{\prime}}{\partial t}=-\nabla p^{\prime}+\rho_{0} g\left(\alpha \theta-\alpha^{\prime} \gamma\right) \hat{\mathbf{k}}-\frac{\mu}{K_{1}} \mathbf{q}^{\prime}+\frac{1}{K_{1}} \mu^{\prime} \nabla^{2} \mathbf{q}^{\prime}+\tilde{\mu} \nabla^{2} \mathbf{q}^{\prime}, & \\
\nabla \cdot \mathbf{q}^{\prime} & =0 \\
A \frac{\partial \theta}{\partial t}+\mathbf{q}^{\prime} \cdot \nabla \theta & =\kappa \nabla^{2} \theta+\beta w \\
A \frac{\partial \gamma}{\partial t}+\mathbf{q}^{\prime} \cdot \nabla \gamma & =\kappa^{\prime} \nabla^{2} \gamma+\beta^{\prime} w
\end{aligned}
$$

where $A=\frac{\left(\rho_{0} C_{0}\right)_{m}}{\left(\rho_{0} C_{0}\right)_{f}}, \kappa^{\prime}=\frac{k^{\prime}}{\left(\rho_{0} C_{0}\right)_{f}} \quad$ and $\kappa=\frac{k}{\left(\rho_{0} C_{0}\right)_{f}}$.

The boundary conditions are

$$
\mathbf{q}^{\prime}=\mathbf{0}, \theta=0, \gamma=0 \quad \text { at } \quad z= \pm \frac{d}{2},
$$

\section{Non-Linear Stability Analysis}

To investigate the non-linear stability analysis, the governing equations (7)-(10) in the non-dimensional form (dropping asterisk) can be written as

$$
\begin{gathered}
\frac{1}{V_{a}} \frac{\partial \mathbf{q}}{\partial t}=-\nabla p+R^{1 / 2} \theta \hat{\mathbf{k}}-\mathbf{q}+\left(F+\tilde{D}_{a}\right) \nabla^{2} \mathbf{q}-\frac{S^{1 / 2}}{L e} \gamma \hat{\mathbf{k}}, \\
\nabla \cdot \mathbf{q}=0, \\
A \frac{\partial \gamma}{\partial t}+\mathbf{q} \cdot \nabla \gamma=\frac{1}{L_{e}} \nabla^{2} \gamma+S^{1 / 2} w, \\
A \frac{\partial \gamma}{\partial t}+\mathbf{q} \cdot \nabla \gamma=\frac{1}{L_{e}} \nabla^{2} \gamma+S^{1 / 2} w,
\end{gathered}
$$

where the following non-dimensional quantities and parameters are introduced:

$$
\begin{gathered}
t^{*}=\frac{\kappa}{d^{2}} t^{\prime}, \mathbf{q}^{*}=\frac{d}{\kappa} \mathbf{q}^{\prime}, \theta^{*}=\frac{R^{1 / 2}}{\beta d} \theta^{\prime}, p^{*}=\frac{K_{1}}{\mu \kappa} p^{\prime}, \\
z^{*}=\frac{1}{d} z, V_{r}=\frac{\tilde{\mu}}{\mu}, V_{a}=\frac{\varepsilon v d^{2}}{\kappa K_{1}}, \\
F=\frac{\mu^{\prime}}{v \rho_{0} d^{2}}, D_{a}=\frac{K_{1}}{d^{2}}, L_{e}=\frac{\kappa}{\kappa^{\prime}}, P_{r}=\frac{v}{\kappa}, S=\frac{g \alpha^{\prime} \beta^{\prime} \rho_{0} d^{2} K_{1}}{\mu \kappa^{\prime}}, \gamma^{*}=\frac{S^{1 / 2}}{\beta^{\prime} d} \gamma \\
R=\frac{g \alpha \beta \rho_{0} K_{1} d^{2}}{\mu \kappa}, \tilde{D}_{a}=\frac{\tilde{\mu} K_{1}}{\mu d^{2}}=V_{r} D_{a} .
\end{gathered}
$$

Here, $R$ is the Rayleigh-Darcy number, which is the product of Darcy number and the usual Rayleigh number for a clear viscous fluid; $\tilde{D}_{a}$ is the Darcy-Brinkman number; $D_{a}$ is the Darcy number; $V_{a}$ is the Vadasz number (as named by Straughan [9]), $F$ is the Couplestress parameter, $S$ is the solute Rayleigh-Darcy number and $L_{e}$ is the Lewis number.

On multiplying (12) by $\mathbf{q}, 14)$ by $\theta$, (15) by $\gamma$ and integrating over $V$, we get (after using equation (11), the boundary conditions and the divergence theorem):

$$
\frac{1}{2 V_{a}} \frac{d\|\mathbf{q}\|^{2}}{d t}=-\|\mathbf{q}\|^{2}-\left(F+\tilde{D}_{a}\right)\|\nabla \mathbf{q}\|^{2}+R^{1 / 2}\langle w \theta\rangle-\frac{S^{1 / 2}}{L_{e}}\langle w \gamma\rangle,
$$

$$
\frac{A}{2} \frac{d\|\theta\|^{2}}{d t}=-\|\nabla \theta\|^{2}+R^{1 / 2}\langle w \theta\rangle,
$$

with $\mathbf{q}^{\prime}, \theta$ and $\gamma$ satisfying the plane tiling periodicity. 


$$
\frac{A}{2} \frac{d\|\gamma\|^{2}}{d t}=-\frac{1}{L_{e}}\|\nabla \gamma\|^{2}+S^{1 / 2}\langle w \gamma\rangle
$$

where $\langle\cdot\rangle$ denotes the integration over $V,\|\cdot\|$ denotes the $L^{2}(V)$ norm and $V$ denotes a typical periodicity cell.

To study the non-linear stability of the basic state (5), an $L^{2}$ energy, $E(t)$, is constructed using equations (17)(19), and the evolution of $E(t)$ is given by

$$
\frac{d E}{d t}=I_{0}-D_{0},
$$

where

$$
\begin{gathered}
E=\frac{A}{2}\|\theta\|^{2}+\frac{\lambda_{1}}{2 V_{a}}\|\mathbf{q}\|^{2}-\frac{\lambda_{2} A}{2}\|\gamma\|^{2}, \\
I_{0}=\left(\lambda_{1}+1\right) R^{1 / 2}\langle w \theta\rangle-\left(\lambda_{2}+\frac{\lambda_{1}}{L_{e}}\right) S^{1 / 2}\langle w \gamma\rangle, \\
D_{0}=\|\nabla \theta\|^{2}+\lambda_{1}\|\mathbf{q}\|^{2}+\lambda_{1}\left(F+\tilde{D}_{a}\right)\|\nabla \mathbf{q}\|^{2}-\frac{\lambda_{2}}{L_{e}}\|\nabla \gamma\|^{2},
\end{gathered}
$$

with $\lambda_{1}$ and $\lambda_{2}$ as two positive coupling parameters.

Here, the negative sign with the $\frac{\lambda_{2} A}{2}\|\gamma\|^{2}$ term in the energy equation (21) shows that energy of the system is consumed due to solute concentration as the system is soluted from below. Now, we take the assumption that the energy consumed due to solute concentration is less than the energy produced due to velocity and temperature. We also assume that the energy dissipated by the solute concentration is less than the energy dissipated by the velocity and temperature. These assumptions will ensure that all the terms on the right-hand side of (21) and (23) are always less than those on the left-hand side of these equations.

We now define,

$$
m=\max _{H} \frac{I_{0}}{D_{0}},
$$

where $H$ is the space of admissible solutions.

Then, we require $m<1$ so that

$$
\frac{d E}{d t} \leq-a_{0} D_{0}
$$

where $a_{0}=1-m(>0)$.

From the Poincaré inequality, we have

$D_{0} \geq \pi^{2}\|\theta\|^{2}+\lambda_{1}\left[1+\pi^{2}\left(F+\tilde{D}_{a}\right)\right]\|\mathbf{q}\|^{2}-\frac{\lambda_{2}}{L_{e}}\|\gamma\|^{2} \geq k^{*} E$

where $k^{*}=2 \pi^{2} \min \left(A, V_{a}^{-1}\right)$.
This gives

$$
\frac{d E}{d t} \leq-a_{0} k^{*} E
$$

implying

$$
E(t) \leq e^{-a_{0} k^{*} t} E(0)
$$

Thus, $E$ decays at least exponentially fast, and non-linear stability is assured for all values of $E(0)$. It is important to note that the result holds for all initial data.

\section{Variational Problem}

Wenow return to equation (24) and use calculus of variation to find the maximum problem at the critical argument $m=1$. The associated Euler-Lagrange equations after taking transformations $\hat{\mathbf{q}}={\sqrt{\lambda_{1}}}_{1} \mathbf{q}$ and $\hat{\gamma}=\sqrt{\lambda}_{2} \gamma$ (dropping caps) are

$$
\begin{gathered}
2\left(F+\tilde{D}_{a}\right) \nabla^{2} \mathbf{q}-2 \mathbf{q}+R^{1 / 2}\left(1+\lambda_{1}\right) \frac{1}{\lambda_{1}^{1 / 2}} \theta \hat{\mathbf{k}}-S^{1 / 2}\left(\lambda_{2}+\frac{\lambda_{1}}{L_{e}}\right) \frac{1}{\lambda_{1}^{1 / 2} \lambda_{2}^{1 / 2}} \gamma \hat{\mathbf{k}}=2 \nabla p, \\
2 \nabla^{2} \theta+R^{1 / 2}\left(1+\lambda_{1}\right) \frac{1}{\lambda_{1}^{1 / 2}} w=0 \\
\frac{2}{L_{e}} \nabla^{2} \gamma+S^{1 / 2}\left(\lambda_{2}+\frac{\lambda_{1}}{L_{e}}\right) \frac{1}{\lambda_{1}^{1 / 2} \lambda_{2}^{1 / 2}} w=0
\end{gathered}
$$

where $p$ is a Lagrange's multiplier introduced, since $\mathbf{q}$ is solenoidal.

On taking curl curl of equation (29) and then taking the third component of the resulting equation, we find

$$
2\left(F+\tilde{D}_{a}\right) \nabla^{4} w-2 \nabla^{2} w+R^{1 / 2}\left(1+\lambda_{1}\right) \frac{1}{\lambda_{1}^{1 / 2}} \nabla_{1}^{2} \theta-S^{1 / 2}\left(\lambda_{2}+\frac{\lambda_{1}}{L_{e}}\right) \frac{1}{\lambda_{1}^{1 / 2} \lambda_{2}^{1 / 2}} \nabla_{1}^{2} \gamma=0 .
$$

Now, we assume a plane tiling form

$$
(w, \theta, \gamma)=[W(z), \Theta(z), \Gamma(z)] g(x, y),
$$

where $\nabla_{1}^{2} g+a^{2} g=0, a$ being the wave number $[9,42]$.

The wave number is found a posteriori to be non-zero; thus, from equations (29)-(31), we see that $W$ and $\Theta$ satisfy

$$
\begin{aligned}
2\left(F+\tilde{D}_{a}\right)\left(D^{2}-a^{2}\right)^{2} W-2\left(D^{2}-a^{2}\right) W- & R^{1 / 2} a^{2}\left(1+\lambda_{1}\right) \frac{1}{\lambda_{1}^{1 / 2}} \Theta \\
+ & S^{1 / 2} a^{2}\left(\lambda_{2}+\frac{\lambda_{1}}{L_{e}}\right) \frac{1}{\lambda_{1}^{1 / 2} \lambda_{2}^{1 / 2}} \gamma=0,
\end{aligned}
$$




$$
\begin{gathered}
\frac{2}{L_{e}}\left(D^{2}-a^{2}\right) \Gamma+S^{1 / 2}\left(\lambda_{2}+\frac{\lambda_{1}}{L_{e}}\right) \frac{1}{\lambda_{1}^{1 / 2}} W=0, \\
\frac{2}{L_{e}}\left(D^{2}-a^{2}\right) \Gamma+S^{1 / 2}\left(\lambda_{2}+\frac{\lambda_{1}}{L_{e}}\right) \frac{1}{\lambda_{1}^{1 / 2} \lambda_{2}^{1 / 2}} W=0 .
\end{gathered}
$$

Thus, the exact solution to the equations (34)-(36) subject to boundary conditions

$W=0, \quad D^{2} W=0, \quad \Theta=0, \Gamma=0$ at $z= \pm \frac{1}{2}$

is written in the form

$W=A_{0} \cos \pi z, \Theta=B_{0} \cos \pi z, \Gamma=C_{0} \cos \pi z$

where $A_{0}, B_{0}$ and $C_{0}$ are constants. Substituting solution (37) in equations (34)-(36), we get the equations involving coefficients of $A_{0}, B_{0}$ and $C_{0}$. For the existence of non-trivial solutions, the determinant of the coefficients of $A_{0}, B_{0}$ and $C_{0}$ must vanish. This determinant on simplification yields

$$
R_{e}=\frac{\frac{4}{L_{e}}(1+x)^{2}\left\{1+\left(F_{1}+\hat{D}_{a}\right)(1+x)\right\}+\frac{x S_{1}}{\lambda_{1} \lambda_{2}}\left(\lambda_{2}+\frac{\lambda_{1}}{L_{e}}\right)^{2}}{x \frac{1}{\lambda_{1} L_{e}}\left(1+\lambda_{1}\right)^{2}},
$$

where

$R_{e}=\frac{R}{\pi^{2}}, \hat{D}_{a}=\pi^{2} \tilde{D}_{a}, x=\frac{a^{2}}{\pi^{2}}, F_{1}=F \pi^{2}$ and $S_{1}=\frac{S}{\pi^{2}}$.

The maximum value of $\lambda_{1}$ and $\lambda_{2}$ is determined by the conditions $\frac{d R_{e}}{d \lambda_{1}}=0$ and $\frac{d R_{e}}{d \lambda_{2}}=0$ and is found to be

$$
\lambda_{1}=1, \lambda_{2}=\frac{1}{L_{e}} .
$$

Using (40) in equation (39), we have

$$
R_{e}=\frac{(1+x)^{2}\left\{1+\left(F_{1}+\hat{D}_{a}\right)(1+x)\right\}}{x}+S_{1} .
$$

We obtain the fluid-based thermal Rayleigh number as

$$
R_{e f}=\frac{R_{e}}{D_{a}}=\frac{(1+x)^{2}\left\{(1+x) V_{r}+\frac{1}{D_{a}}\left\{1+F_{1}(1+x)\right\}\right\}}{x}+S_{f} .
$$

As a function of $x, R_{e f}$ given by equation (42) attains its minimum when

$$
P_{3} x^{3}+P_{2} x^{2}+P_{0}=0
$$

where

$$
P_{3}=2\left(V_{r}+\frac{F_{1}}{D_{a}}\right), P_{2}=\left[\frac{1}{D_{a}}+\left(V_{r}+\frac{F_{1}}{D_{a}}\right)\right] \text { and } P_{0}=-\left[V_{r}+\left(\frac{1+F_{1}}{D_{a}}\right)\right] \text {. }
$$

The thermal Rayleigh number $R_{e f}$ is minimized with respect to $x$, and we use the Newton-Raphson iterative scheme to obtain the value of critical wave number and the corresponding critical thermal Rayleigh number $R_{c e f}$ (see Tables 1-3).

For analyzing the linear instability results, we return to the perturbed equations (7)-(10), neglecting the nonlinear terms. We again perform the standard stationary normal mode analysis and look for the solution of these equations in the form (38). The boundary conditions in the present case are same, i.e. (37) (here, the thermal Rayleigh number).

$$
R_{\ell f}=\frac{(1+x)^{2}\left\{(1+x) V_{r}+\frac{1}{D_{a}}\left\{1+F_{1}(1+x)\right\}\right\}}{x}+S_{f}=R_{e f}
$$

In the absence of solute ( $S_{f}=0$ ), this further simplifies to

$$
R_{\ell f}=\frac{(1+x)^{2}\left\{(1+x) V_{r}+\frac{1}{D_{a}}\left\{1+F_{1}(1+x)\right\}\right\}}{x}=R_{e f}
$$

i.e., in both the case, the linear instability boundary $\equiv$ the non-linear stability boundary, and so no subcritical instabilities are possible for the case of couple-stress fluid. This result is equivalent to the result given by Joseph $[4,5]$ for the standard Bénard problem.

\section{Discussion Of Results And Conclusion}

The critical wave numbers $x_{c \ell \text { and }} x_{c e}$ and critical thermal Rayleigh number $\mathrm{R}_{e f}=R_{c e f}$ depends on the parameters $V_{r}, F_{1}, S_{f}$ and $D_{a}$. The variation in $x_{c}$ and $R_{c e f}$ with the variation in $F_{1}$ is given in Table 1, that with the variation in $D_{a}$ is given in Table 2 and that with the variation in $S_{f}$ is given in Table 3, and the 
Table 1: The variation in the fluid-based critical thermal Rayleigh number $R_{\text {cef }}$ with the couple-stress parameter $F_{1}$ for $D_{a}=0.02, V_{r}=1$ and $S_{f}=100$.

\begin{tabular}{lll}
\hline $\boldsymbol{F}_{1}$ & $\boldsymbol{x}_{c e}$ & $\boldsymbol{R}_{c e f}$ \\
\hline 01 & 0.9636 & 307.90 \\
2 & 0.6154 & 661.40 \\
3 & 0.5682 & 1001.9 \\
4 & 0.5483 & 1340.8 \\
5 & 0.5374 & 1679.0 \\
6 & 0.5305 & 2017.0 \\
7 & 0.5258 & 23537 \\
8 & 0.5223 & 2696.0 \\
9 & 0.5197 & 3030.3 \\
10 & 0.5176 & 3367.9 \\
& 0.5159 & 3705.6 \\
\hline
\end{tabular}

Table 2: The variation in the fluid-based critical thermal Rayleigh number $R_{c e f}$ with the couple-stress parameter $\left(D_{a}\right)$ for $F_{1}=2, V_{r}=1$ and $S_{f}=100$.

\begin{tabular}{lll}
\hline $\boldsymbol{D}_{a}$ & $\boldsymbol{x}_{c e}$ & $\boldsymbol{R}_{\text {cef }}$ \\
\hline 0.01 & 0.5684 & 1897.1 \\
0.02 & 0.5682 & 1001.9 \\
0.03 & 0.5679 & 7017 \\
0.04 & 0.5676 & 5532 \\
0.05 & 0.5673 & 4636 \\
0.06 & 0.5671 & 405.2 \\
0.07 & 0.5668 & 365.0 \\
0.08 & 0.5665 & 330.6 \\
0.09 & 0.5663 & 305.7 \\
0.10 & 0.5660 & 285.8 \\
0.11 & 0.5667 & 269.5 \\
\hline
\end{tabular}

Table 3: The variation in the fluid-based critical thermal Rayleigh number $R_{c e f}$ with the solute gradient $\left(S_{p}\right)$ for $D_{a}, V_{r}=1$.

\begin{tabular}{llll}
\hline $\boldsymbol{S}$ & $\boldsymbol{R}_{c e f}\left(\begin{array}{l}F_{1}=1 \\
x_{c e}=0.6164\end{array}\right)$ & $\boldsymbol{R}_{c e f}\left(\begin{array}{l}F_{1}=2 \\
x_{c e}=0.5682\end{array}\right)$ & $\boldsymbol{R}_{c e f}\left(\begin{array}{l}F_{1}=3 \\
x_{c e}=0.5483\end{array}\right)$ \\
\hline 100 & 661.4 & 1001.9 & 1340.8 \\
200 & 761.4 & 1101.9 & 1440.8 \\
300 & 861.4 & 1201.9 & 1540.8 \\
400 & 961.4 & 1301.9 & 1640.8 \\
500 & 1061.4 & 1401.9 & 1740.8 \\
600 & 1161.4 & 1501.9 & 1840.8 \\
700 & 271.4 & 1601.9 & 1940.8 \\
800 & 1361.4 & 1701.9 & 2040.8 \\
900 & 1461.4 & 1801.9 & 2140.8 \\
\hline
\end{tabular}

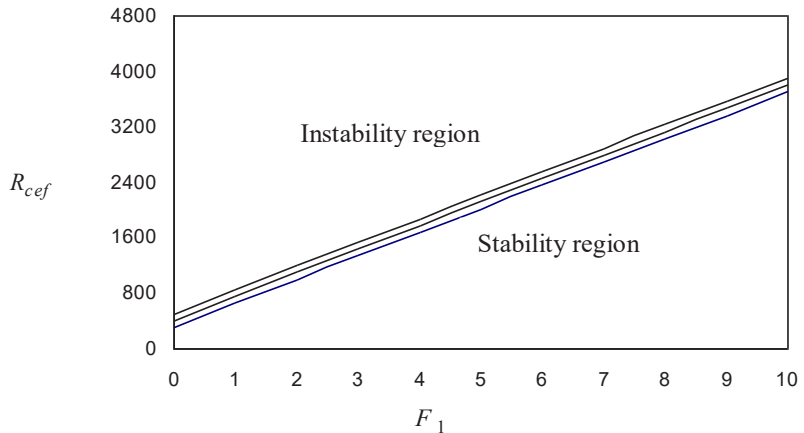

Figure 2: The variation in the critical thermal Rayleigh number $R_{c e f}$ with the couple-stress parameter $F_{1}$.

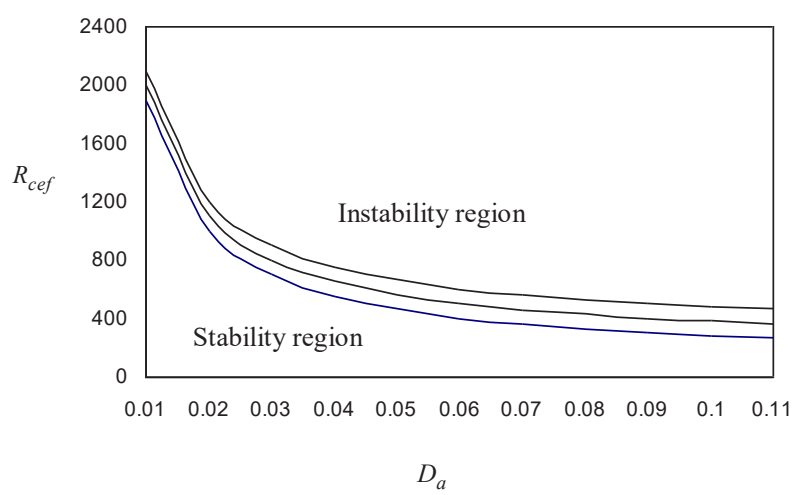

Figure 3: The variation in the critical thermal Rayleigh number $R_{c e f}$ with the couple -stress parameter $D_{a}$.

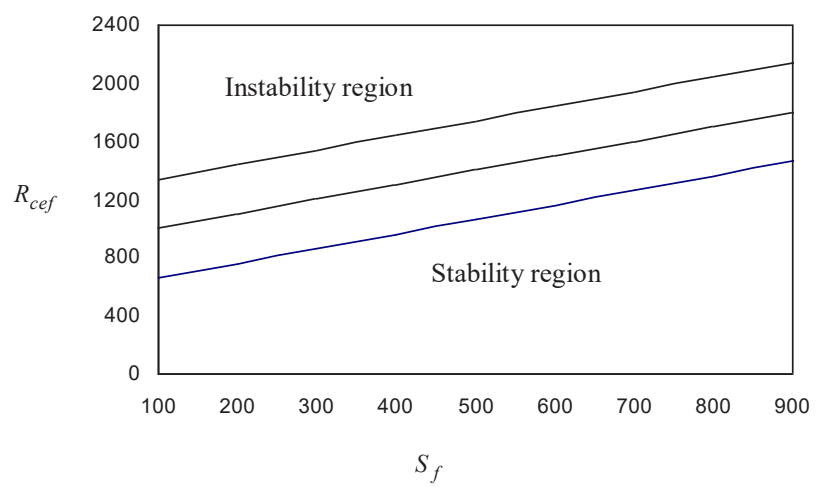

Figure 4: The variation in the critical thermal Rayleigh number $R_{\text {cef }}$ with the solute gradient $S_{f}$.

results are further illustrated in Figs. 2-4, which represent the plot of critical thermal Rayleigh number $R_{c e f}$ versus the parameter $F_{1}, D_{a}$ and $S_{f}$, respectively. Figure 2 indicates that the parameter $F_{1}$ has the stabilizing effect on convection because as $F_{1}$ increases, the value of $R_{c e f}$ increases. We also note that the value of critical thermal Rayleigh number remains the same for both the theories 
(linear theory and non-linear theory) and no subcritical instabilities are possible.

This conclusion is further strengthened in Fig. 3, which shows the variation in $R_{c e f}$ with Darcy number $D_{a}$ at $F$ $=2, V_{r}=1$. Here, an increase in $D_{a}$ leads to a decrease in $R_{c e f}$, rendering the system prone to instability. Figure 4 indicates that the solute gradient $S_{f}$ has a stabilizing effect because with the increase in $S_{f}$, the values of $R_{c e f}$ also increase. Here, two diffusing components heat and salt are present that produce the density differences required to derive the motion. The components make opposing contributions to the vertical density gradient as motion is encouraged due to heating and solute acts to prevent motion through convection overturning. Thus, these two physical effects are competing against each other. We also note that the value of critical thermal Rayleigh number remains the same for both the theories (linear theory and non-linear theory) and no subcritical instabilities are possible. In other words, medium permeability destabilizes the flow. To investigate our result, we must review the results and its physical explanation. When the fluid layer is assumed to be flowing through an isotropic and homogenous porous medium, then the medium permeability has a destabilizing effect. This is because, as medium permeability increases, the void space increases, and as a result of this, the flow quantities perpendicular to the planes will clearly be increased. Thus, an increase in heat transfer is responsible for early onset of convection. Hence, an increase in $D_{a}$ leads to a decrease in $R_{c e f}$.

The principal conclusion from the above analysis is as follows:

- The result we establish is that boundaries of nonlinear stability and linear instability analyses coincide with each other. So, no subcritical instabilities are possible.

- The couple stress has the tendency to slow down the motion of the fluid in the boundary layer, thus reducing the heat transfer from bottom to top. The a decrease in heat transfer is responsible for delaying the onset of convection. Thus, the couple-stress parameter $F_{1}$ promotes stabilization.

- The medium permeability is found to have destabilizing effect on the system.

- It is observed that solute gradient delays the onset of convection and thus has a stabilizing effect on the system.

\section{References}

[1] Joseph, D.D. (1976). Stability of Fluid Motions. Vols. I-II, Springer, Berlin.

[2] Orr, W. MCF. (1907). Stability or instability of the steady motions of a perfect liquid. Proceedings of the Royal Irish Academy, A 37, 9.

[3] Serrin, J. (1959). On the stability of viscous fluid motions. Archive for Rational Mechanics and Analysis, 3, 1.

[4] Joseph, D.D. (1965). On the stability of the Boussinesq equations. Archive for Rational Mechanics and Analysis, 20, 59.

[5] Joseph, D.D. (1966). Nonlinear stability of the Boussinesq equations by the method of energy. Archive for Rational Mechanics and Analysis, 22, 163.

[6] Galdi, G.P., Padula, M. (1990). A new approach to energy theory in the stability of fluid motion. Archive for Rational Mechanics and Analysis, 110, 187.

[7] Straughan, B., Explosive Instabilities in Mechanics, Springer, Berlin, 1998.

[8] Straughan, B. (2004). The Energy Method, Stability, and Nonlinear Convection. Springer-Verlag, New York.

[9] Straughan, B. (2005). A sharp nonlinear stability threshold in rotating porous convection. Proceedings of the Royal Society of London Series A, 457, 87.

[10] Kaloni, P.N., Qiao, Z. (1997). Non-linear stability of convection in a porous medium with inclined temperature gradient. International Journal of Heat and Mass Transfer, 40, 1611.

[11] Kaloni, P.N., Qiao, Z. (1997). Nonlinear convection with inclined temperature gradient and horizontal mass flow. International Journal of Engineering Science, 35, 299.

[12] Kaloni, P.N., Qiao Z. (2001). Non-linear convection in a porous medium with inclined temperature gradient and variable gravity effects. International Journal of Heat and Mass Transfer, 44, 1585.

[13] Guo, J., Kaloni, P.N. (1995). Nonlinear stability problem of a rotating doubly diffusive porous layer. Journal of Mathematical Analysis and Applications, 190, 373.

[14] Guo, J., Qin, Y., Kaloni, P.N. (1994). Non-linear stability problem of a rotating doubly diffusive fluid layer. International Journal of Engineering Science, 32, 1207.

[15] Payne, L.E., Straughan, B. (2000). Unconditional nonlinear stability in temperature - dependent viscosity flow in a porous medium. Studies in Applied Mathematics, 105, 59.

[16] Stokes, V.K. (1966). Couple stresses in fluids. The Physics of Fluids, 9, 1709.

[17] Ramanaiah, G., Sarkar, P. (1979). Slider bearings lubricated by fluids with couple stress. Wear, 52, 27.

[18] Sharma R. C., Thakur K. D. (2000). On couple-stress fluid heated from below in porous medium in hydromagnetics. Czechoslovak Journal of Physics, 50, 753.

[19] Sharma R. C., Sunil, Pal M. (2000). On couple-stress fluid heated from below in porous medium in presence of rotation. Applied Mechanical Engineering, 5(4), 883.

[20] Sunil, Mahajan A. (2008a). A nonlinear stability analysis for magnetized ferrofluid heated from below. Proceedings of the Royal Society A, 464, 83.

[21] Sunil, Mahajan A. (2008b). A nonlinear stability analysis for rotating magnetized ferrofluid heated from below. Applied Mathematics and Computation, 204, 299. 
[22] Sunil, Mahajan A. (2008c). A nonlinear stability analysis of a double-diffusive magnetized ferrofluid. Zeitschrift für Naturforschung, 63a, 797.

[23] Sunil, Mahajan A. (2008d). A nonlinear stability analysis in a double-diffusive magnetized ferrofluid layer saturating a porous medium. Journal of Geophysics and Engineering, 5(3), 311.

[24] Sunil, Choudhary S., Bharti P. (2013). Global stability for thermal convection in a couple-stress fluid with temperature and pressure dependent viscosity. Studia Geotechnica et Mechanica, 35(3), 85.

[25] Sunil, Choudhary S., Bharti P. (2012). Global stability for thermal convection in a couple-stress fluid saturating a porous medium with temperature and pressure dependent-dependent viscosity. International Journal of Applied Mechanics and Engineering, 17(2), 583.

[26] Hsu C. H., Lin J. R., Chiang H.L. (2003). Combined effects of couple stresses and surface roughness on the lubrication of short journal bearings. Industrial Lubrication and Tribology, 55, 233.

[27] Lahmar, M. (2005). Elastohydrodynamic analysis of doublelayered journal bearings lubricated with couple-stress fluids. Proceedings of the Institution of Mechanical Engineers, Part J: Journal of Engineering Tribology, 219, 145.

[28] Nield, D.A., Bejan A. (2006). Convection in Porous Media, Springer, New York.

[29] Veronis, G. (1968). Effect of a stabilizing gradient of solute on thermal convection. Journal of Fluid Mechanics, 34, 315.

[30] Banies, P.G., Gill A. E. (1969). On thermohaline convection with linear gradients. Journal of Fluid Mechanics, 37, 289.

[31] Joseph, D.D. (1970). Global stability of the conduction-diffusion solution. Archive for Rational Mechanics and Analysis, 36, 285.

[32] Griffiths, R.W. (1981). Layered double-diffusive convection in porous media. Journal of Fluid Mechanics, 102, 221.

[33] Sunil, Sharma, D., Sharma, R.C. (2004). Effect of rotation on ferromagnetic fluid heated and soluted from below saturating a porous medium. Journal of Geophysics and Engineering, 1, 116.

[34] Sunil, Sharma, A., Sharma, R.C. (2006). Effect of dust particles on ferrofluid heated and soluted from below. International Journal of Thermal Sciences, 45, 347.

[35] Sunil, Sharma, A., Bharti, P.K., Shandil, R.G. (2007). Linear stability of double-diffusive convection in a micropolar ferromagnetic fluid saturating a porous medium. International Journal of Mechanical Sciences, 49, 1047.

[36] Sunil, Sharma, P., Mahajan, A. (2009). A nonlinear stability analysis of a rotating double-diffusive magnetized ferrofluid saturating a porous medium. Heat Transfer Research, 40, 351.

[37] Sunil, Sharma, P., Mahajan, A. (2010). Onset of DarcyBrinkman double-diffusive convection in a magnetized ferrofluid layer using a thermal non-equilibrium model: a nonlinear stability analysis. Journal of Geophysics and Engineering, 7, 417.

[38] Mahajan, A., Nandal, R. (2017). On the stability of penetrative convection in a couple-stress fluid. International Journal of Applied and Computational Mathematics, 3(4), 3745-3758.

[39] Nandal, R., Mahajan, A. (2018). Penetrative convection in couple-stress fluid via internal heat source/sink with the boundary effects. Journal of Non-Newtonian Fluid Mechanics, 260, 133-141.
[40] Qin, Y., Kaloni, P.N. (1995). Nonlinear stability problem of a rotating porous layer. Quarterly of Applied Mathematics, 53, 129.

[41] Finlayson, B.A. (1970). Convective instability of ferromagnetic fluids. Journal of Fluid Mechanics, 40, 753.

[42] Chandrasekhar, S. (1981). Hydrodynamic and Hydromagnetic Stability, Dover, New York. 